\title{
A new model of cell dynamics in Acute Myeloid Leukemia involving distributed delays $^{\star}$
}

\author{
J. L. Avila* C. Bonnet* J. Clairambault ${ }^{* *}$ H. Özbay*** \\ S. I. Niculescu ${ }^{* * * *}$ F. Merhi ${ }^{\dagger}$ R. Tang ${ }^{\dagger}$ J.P. Marie ${ }^{\dagger}$ \\ * INRIA Saclay - Île-de-France, Equipe DISCO, LSS - SUPELEC, 3 \\ rue Joliot Curie, 91192 Gif-sur-Yvette, Cedex, France. \\ ** INRIA Paris-Rocquencourt, Domaine de Voluceau, B.P. 105, 78153 \\ Le Chesney, Cedex, France, and INSERM team U 776 "Biological \\ Rhythms and Cancers", Hôpital Paul-Brousse, 14 Av. \\ Paul-Vaillant-Couturier, 94807 Villejuif, Cedex, France. \\ *** Dept. of Electrical and Electronics Eng., Bilkent University, \\ Ankara, 06800, Turkey. \\ **** L2S (UMR CNRS 8506), CNRS-Supélec, 3 rue Joliot Curie, \\ 91192, Gif-sur-Yvette, France \\ † Tumorothèque d'Hématologie, Hôpital Saint-Antoine, AP-HP, 184 \\ rue du Faubourg Saint Antoine, 75571 Paris cedex 12, France; \\ INSERM U872, Université Pierre et Marie Curie, Centre de \\ Recherche des Cordeliers, 15 Rue de l'Ecole de Médecine, 75270 Paris \\ Cedex 06, France.
}

\begin{abstract}
:
In this paper we propose a refined model for the dynamical cell behavior in Acute Myeloid Leukemia (AML) compared to (Özbay et al, 2012) and (Adimy et al, 2008). We separate the cell growth phase into a sequence of several sub-compartments. Then, with the help of the method of characteristics, we show that the overall dynamical system of equations can be reduced to two coupled nonlinear equations with four internal sub-systems involving distributed delays.
\end{abstract}

Keywords: Modelling, PDE, Delay, Medical applications, nonlinear models.

\section{INTRODUCTION}

In this paper, we propose a new model of cell dynamics in Acute Myeloid Leukemia (AML), a disease for which clinical progress has been quite slow in the last forty years, Rowe (2008). Our aim is to design continuous mathematical models in order to better understand its dynamical behavior and ultimately improve its treatment.

The formation and maturation of blood cells is called hematopoiesis. Blood cells mature in the bone marrow from hematopoietic stem cells (HSCs) until normally fully differentiated cells of different lineages are released in the general blood circulation. Various dynamical models have been proposed and studied in the literature for the hematopoietic processes, see e.g. recent works of Adimy et al. (2008), Dingli and Pacheco (2010), Foley and Mackey (2009) Niculescu et al. (2010) and their references. In normal hematopoiesis, HSCs proliferate, either self-renewing or differentiating. The proliferation process in cell populations relies on the cell division cycle consisting of four phases (phase $G_{1}$, phase $S$, phase $G_{2}$ and phase $M$ ) at the end of which cell division occurs. Each

* This work was supported by the DIGITEO Project ALMA partly funded by the Région Île-de-France, France dividing (mother) cell gives birth to two daughter cells, of possibly different types: either cells that have the same biological properties - in particular stuck at the same differentiation stage - as the mother cell (self-renewal) or other cells, more advanced in the maturation process (the production of progenitors at cell division being called differentiation). From HSCs through this differentiation process are produced progenitors, that are the precursors of three blood lineages (red blood cells, white blood cells or platelets). We are interested here only in the myeloid lineage among white blood cells, which we will consider at the cell population level, structured in age with respect to cell cycle phases. Physiologically, it is only when they have reached full maturity that hematopoietic cells are released in the general blood circulation. It may occur that one genetic alteration appears in a hematopoietic stem cell, escapes the various physiological controls and is transmitted by subsequent divisions to daughter cells to eventually yield a leukemia. AML combines at least two molecular events: a blockade of the differentiation and an advantage of the proliferation (in particular progenitors may self-renew). This blockade of differentiation in AML results in an overflow of immature and inefficient cells firstly in the bone marrow, and eventually in blood. 
One of the first mathematical models on hematopoiesis was proposed by Mackey (1978) at the end of the 1970's. This model consists of a system of differential equations describing HSC's dynamics, considering a resting (or quiescent) phase and a proliferative phase. A few years ago, in order to take the differentiation process into account, a PDE based model including several compartments connected in series was proposed by Adimy et al. (2008). From the system theory point of view this model is a distributed delay system with static nonlinearity. For the analysis of this model (equilibrium analysis and stability of the linearized as well as nonlinear system) see the recent papers Adimy et al. (2010), Ozbay et al (2010), Ozbay et al. (2012) and their references.

The aim of this paper is to modify and enrich the model of Adimy et al. (2008) in the following sense:

- the self-renewal phenomenon is written in two parts where fast and slow dynamics are separated (this gives us two static nonlinearities in the system), and

- the dynamical behavior of the proliferating cells is separated into four phases (namely the phases $G_{1}, S$, $G_{2}$ and $\left.M\right)$.

Ultimately, this refined model will help us better evaluate a new therapy strategy which acts on phase $S$.

The paper is organized as follows. In Section 2, we present the PDE based model of cell dynamics. In Section 3, we reduce the model into two coupled nonlinear differential equations involving four distributed delay terms. The equilibrium and stability analysis of this new system can be performed by using techniques similar to the ones employed in Ozbay et al. (2012); in the final version of the paper we will include preliminary results along this direction, as well as numerical simulations.

\section{MATHEMATICAL MODEL OF AML}

Let us consider two cell sub populations of immature cells, proliferating (divided in $G_{1}, S, G_{2}$ and $M$ phases) and quiescent (in phase $G_{0}$ ) cells, at each stage of the compartmental model discussed in Section 1. We denote by $p_{i}(t, a), l_{i}(t, a), n_{i}(t, a), m_{i}(t, a)$ and $r_{i}(t, a)$ the cell populations of the $G_{1}, S, G_{2}, M$ and $G_{0}$ phases, respectively, of the $i$-th generation of immature cells, with age $a \geq 0$ at time $t \geq 0$. We have assumed that the dynamics of the cell population are governed by the following system of partial differential equations

$$
\begin{cases}\frac{\partial p_{i}}{\partial t}+\frac{\partial p_{i}}{\partial a}=-\left(\gamma_{i}^{1}+g_{i}^{p}(a)\right) p_{i}, & 0<a<\tau_{i}^{1}, t>0 \\ \frac{\partial l_{i}}{\partial t}+\frac{\partial l_{i}}{\partial a}=-\left(\gamma_{i}^{2}+g_{i}^{l}(a)\right) s_{i}, & 0<a<\tau_{1}^{2}, t>0 \\ \frac{\partial n_{i}}{\partial t}+\frac{\partial n_{i}}{\partial a}=-\left(\gamma_{i}^{3}+g_{i}^{n}(a)\right) n_{i}, & 0<a<\tau_{i}^{3}, t>0 \\ \frac{\partial m_{i}}{\partial t}+\frac{\partial m_{i}}{\partial a}=-\left(\gamma_{i}^{4}+g_{i}^{m}(a)\right) m_{i}, & 0<a<\tau_{i}^{4}, t>0 \\ \frac{\partial r_{i}}{\partial t}+\frac{\partial r_{i}}{\partial a}=-\left(\delta_{i}+\beta_{i}\right) r_{i}, & a>0, t>0\end{cases}
$$

where the death rate in the resting phase is $\delta_{i} \in \mathbb{R}_{+}$, the reintroduction function from the resting subpopulation into the proliferative subpopulation is $\beta_{i}$, the death rates in the $G_{1}, S, G_{2}$ and $M$ phases are $\gamma_{i}^{1}, \gamma_{i}^{2}, \gamma_{i}^{3}$ and $\gamma_{i}^{4}$ respectively; the time elapsed in the $G_{1}, S, G_{2}$ and $M$ phases are $\tau_{i}^{1}$, $\tau_{i}^{2}, \tau_{i}^{3}$ and $\tau_{i}^{4}$, respectively; and, the division rates of the phases $G_{1}, S, G_{2}$ and $M$ phases are $g_{i}^{p}(a), g_{i}^{l}(a), g_{i}^{n}(a)$ and $g_{i}^{m}(a)$ respectively.

Here only the death rate is included and the birth rate is not involved in the equation because, when individuals are born at $a=0$, they are introduced into the population through the boundary (renewal) condition. The introduction rate $\beta_{i}$ is supposed to depend upon the total population of resting cells, denoted by $x_{i}(t)$, where

$$
x_{i}(t):=\int_{0}^{+\infty} r_{i}(t, a) d a .
$$

We also consider a new phase called $\tilde{G}_{0}$ between the exit of the $M$ phase and the beginning of the $G_{1}$ phase. The number of cells of this new phase is $\tilde{r}_{i}(t, a)$ and satisfies its own transport equation

$$
\frac{\partial \tilde{r}_{i}}{\partial t}+\frac{\partial \tilde{r}_{i}}{\partial a}=-\tilde{\beta}_{i}\left(\tilde{x}_{i}(t)\right) \tilde{r}_{i} \quad a>0, \quad t>0,
$$

where the long term dynamics of the fast self-renewal is $\tilde{x}_{i}(t):=\int_{0}^{+\infty} \tilde{r}_{i}(t, a) d a$. The necessity to model the dynamics of the $\tilde{G}_{0}$ phase is because the behavior of AML has a fast self renewal term at the end of the $M$ phase.

A schematic representation of the compartmental model considered is shown in Figure 1.

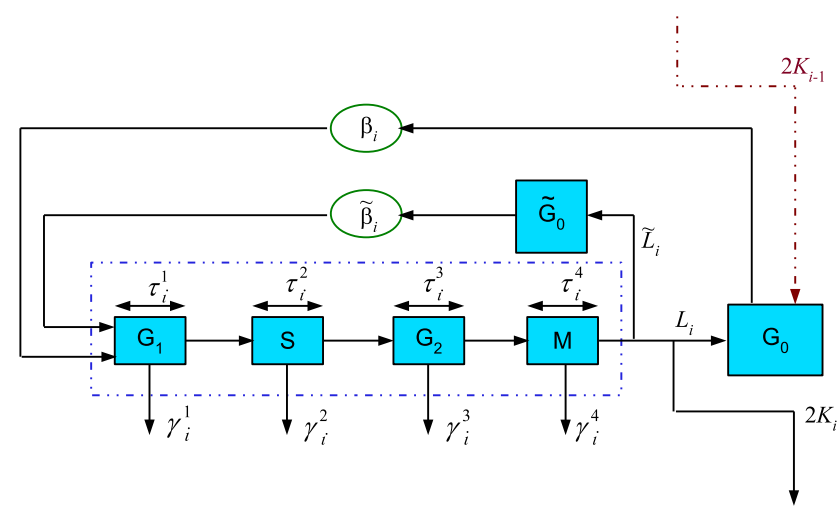

Fig. 1. A refined model of AML cell dynamics

Boundary conditions associated with (1) and (3) are given by 


$$
\left\{\begin{array}{l}
p_{i}(t, a=0)=\beta_{i}\left(x_{i}(t)\right) x_{i}(t)+\tilde{\beta}_{i}\left(\tilde{x}_{i}(t)\right) \tilde{x}_{i}(t), \\
l_{i}(t, a=0)=\int_{0}^{\tau_{i}^{1}} g_{i}^{p}(a) p_{i}(t, a) d a, \\
n_{i}(t, a=0)=\int_{0}^{\tau_{i}^{2}} g_{i}^{l}(a) l_{i}(t, a) d a, \\
m_{i}(t, a=0)=\int_{0}^{\tau_{i}^{3}} g_{i}^{n}(a) n_{i}(t, a) d a \\
r_{i}(t, a=0)=L_{i} \int_{0}^{\tau_{i}^{4}} g_{i}^{m}(a) m_{i}(t, a) d a \\
+2 K_{i-1} \int_{0}^{\tau_{i-1}^{4}} g_{i-1}^{m}(a) m_{i-1}(t, a) d a \\
\tilde{r}_{i}(t, a=0)=\tilde{L}_{i} \int_{0}^{\tau_{4}} g_{i}^{m}(a) m_{i}(t, a) d a .
\end{array}\right.
$$

where $L_{i}:=2 \sigma_{i}\left(1-K_{i}\right), \quad \tilde{L}_{i}:=2\left(1-\sigma_{i}\right)\left(1-K_{i}\right)$. The initial age-distribution of the populations of (1) and (3) are nonnegative functions of age $a$; the functions are assumed to be known: $p_{i}(t=0, a)=p_{i}^{0}(a)$, $l_{i}(t=0, a)=l_{i}^{0}(a), n_{i}(t=0, a)=n_{i}^{0}(a), m_{i}(t=0, a)=$ $m_{i}^{0}(a), r_{i}(t=0, a)=r_{i}^{0}(a)$ and $\tilde{r}_{i}(t=0, a)=\tilde{r}_{i}^{0}(a)$.

The division rates $g_{i}^{p}(a), g_{i}^{l}(a), g_{i}^{n}(a)$ and $g_{i}^{m}(a)$ are assumed to be continuous functions such that $\int_{0}^{\tau_{i}^{1}} g_{i}^{p}(a) d a=$ $+\infty, \int_{0}^{\tau_{i}^{2}} g_{i}^{l}(a) d a=+\infty, \int_{0}^{\tau_{i}^{3}} g_{i}^{n}(a) d a=+\infty$ and $\int_{0}^{\tau_{i}^{4}} g_{i}^{m}(a) d a=+\infty$.

We also assume that

$$
\lim _{a \rightarrow+\infty} r_{i}(t, a)=0
$$

and

$$
\lim _{a \rightarrow+\infty} \tilde{r}_{i}(t, a)=0 .
$$

\section{MODEL TRANSFORMATION}

Using the method of characteristics (see e.g Perthame $(2007)$ ), one easily obtains an explicit formulation for $p_{i}(t, a), l_{i}(t, a), n_{i}(t, a)$ and $m_{i}(t, a)$ given by

$$
\begin{aligned}
& p_{i}(t, a)= \begin{cases}p_{i}^{0}(a-t) e^{-\int_{a-t}^{a}\left(\gamma_{i}^{1}+g_{i}^{p}(w)\right) d w}, & \text { if } t \leq a, \\
p_{i}(t-a, 0) e^{-\int_{0}^{a}\left(\gamma_{i}^{1}+g_{i}^{p}(w)\right) d w} & \text { if } t>a,\end{cases} \\
& l_{i}(t, a)= \begin{cases}l_{i}^{0}(a-t) e^{-\int_{a-t}^{a}\left(\gamma_{i}^{2}+g_{i}^{l}(w)\right) d w}, & \text { if } t \leq a, \\
l_{i}(t-a, 0) e^{-\int_{0}^{a}\left(\gamma_{i}^{2}+g_{i}^{l}(w)\right) d w} & \text { if } t>a,\end{cases} \\
& n_{i}(t, a)= \begin{cases}n_{i}^{0}(a-t) e^{-\int_{a-t}^{a}\left(\gamma_{i}^{3}+g_{i}^{n}(w)\right) d w}, & \text { if } t \leq a, \\
n_{i}(t-a, 0) e^{-\int_{0}^{a}\left(\gamma_{1}^{3}+g_{i}^{n}(w)\right) d w} & \text { if } t>a,\end{cases}
\end{aligned}
$$

$$
m_{i}(t, a)= \begin{cases}m_{i}^{0}(a-t) e^{-\int_{a-t}^{a}\left(\gamma_{i}^{4}+g_{i}^{m}(w)\right) d w}, & \text { if } t \leq a, \\ m_{i}(t-a, 0) e^{-\int_{0}^{a}\left(\gamma_{i}^{4}+g_{i}^{m}(w)\right) d w} & \text { if } t>a,\end{cases}
$$

with

$$
\begin{aligned}
p_{i}(t-a, 0)= & \beta_{i}\left(x_{i}(t-a)\right) x_{i}(t-a) \\
& +\tilde{\beta}_{i}\left(\tilde{x}_{i}(t-a)\right) \tilde{x}_{i}(t-a), \\
l_{i}(t-a, 0)= & \int_{0}^{\tau_{i}^{1}} g_{i}^{p}\left(\theta_{1}\right) p_{i}\left(t-a, \theta_{1}\right) d \theta_{1}, \\
n_{i}(t-a, a)= & \int_{0}^{\tau_{i}^{2}} g_{i}^{l}\left(\theta_{2}\right) l_{i}\left(t-a, \theta_{2}\right) d \theta_{2}, \\
m_{i}(t-a, a)= & \int_{0}^{\tau_{i}^{3}} g_{i}^{n}\left(\theta_{3}\right) n_{i}\left(t-a, \theta_{3}\right) d \theta_{3} .
\end{aligned}
$$

Only the solutions $t \geq a$ are considered for the density cells $p_{i}(t, a), l_{i}(t, a), n_{i}(t, a)$ and $m_{i}(t, a)$ because we are mainly interested in the long time behaviour of the populations; namely, the behaviour of these phases is described by the second term of (4), (5), (6), (7) and the following initial conditions

$p_{i}(t-a, 0)=\beta_{i}\left(\Delta^{a} x_{i}(t)\right) \Delta^{a} x_{i}(t)+\tilde{\beta}_{i}\left(\Delta^{a} \tilde{x}_{i}(t)\right) \Delta^{a} \tilde{x}_{i}(t)$

$$
\begin{aligned}
l_{i}(t-a, 0)= & \int_{0}^{\tau_{i}^{1}}\left(\beta_{i}\left(\Delta^{a+\theta_{1}} x_{i}(t)\right) \Delta^{a+\theta_{1}} x_{i}(t)\right. \\
& \left.+\tilde{\beta}_{i}\left(\Delta^{a+\theta_{1}} \tilde{x}_{i}(t)\right) \Delta^{a+\theta_{1}} \tilde{x}_{i}(t)\right) \\
& \cdot f_{i}^{p}\left(\theta_{1}\right) e^{-\gamma_{1}^{i} \theta_{1}} d \theta_{1}
\end{aligned}
$$

$$
\begin{aligned}
n_{i}(t-a, 0)= & \int_{0}^{\tau_{i}^{2}}\left(\int _ { 0 } ^ { \tau _ { i } ^ { 1 } } \left(\beta_{i}\left(\Delta^{a+\theta_{1}+\theta_{2}} x_{i}(t)\right)\right.\right. \\
& \cdot \Delta^{a+\theta_{1}+\theta_{2}} x_{i}+\tilde{\beta}_{i}\left(\Delta^{a+\theta_{1}+\theta_{2}} \tilde{x}_{i}(t)\right) \\
& \left.\left.\cdot \Delta^{a+\theta_{1}+\theta_{2}} \tilde{x}_{i}(t)\right) f_{i}^{p}\left(\theta_{1}\right) e^{-\gamma_{1}^{i} \theta_{1}} d \theta_{1}\right) \\
& \cdot f_{i}^{l}\left(\theta_{2}\right) e^{-\gamma_{i}^{2} \theta_{2}} d \theta_{2}
\end{aligned}
$$

$$
\begin{aligned}
m_{i}(t-a, 0)= & \int_{0}^{\tau_{i}^{3}}\left[\int _ { 0 } ^ { \tau _ { i } ^ { 2 } } \left(\int _ { 0 } ^ { \tau _ { i } ^ { 1 } } \left(\beta_{i}\left(\Delta^{a+\theta_{1}+\theta_{2}+\theta_{3}} x_{i}(t)\right)\right.\right.\right. \\
& \cdot \Delta^{a+\theta_{1}+\theta_{2}+\theta_{3}} x_{i}(t)+\tilde{\beta}_{i}\left(\Delta^{a+\theta_{1}+\theta_{2}+\theta_{3}} \tilde{x}_{i}(t)\right) \\
& \left.\cdot \Delta^{a+\theta_{1}+\theta_{2}+\theta_{3}} \tilde{x}_{i}(t)\right) \\
& \left.\left.\cdot f_{i}^{p}\left(\theta_{1}\right) e^{-\gamma_{1}^{i} \theta_{1}} d \theta_{1}\right) f_{i}^{l}\left(\theta_{2}\right) e^{-\gamma_{i}^{2} \theta_{2}} d \theta_{2}\right] \\
& \cdot f_{i}^{n}\left(\theta_{3}\right) e^{-\gamma_{i}^{3} \theta_{3}} d \theta_{3}
\end{aligned}
$$

where

$$
\begin{aligned}
f_{i}^{p}(t) & =g_{i}^{p}(t) e^{-\int_{0}^{t} g_{i}^{p}(w) d w} \quad \text { if } 0<t<\tau_{1} \\
& =0 \quad \text { otherwise, }
\end{aligned}
$$$$
f_{i}^{l}(t)=g_{i}^{l}(t) e^{-\int_{0}^{t} g_{i}^{l}(w) d w} \quad \text { if } 0<t<\tau_{2}
$$$$
=0 \text { otherwise, }
$$ 


$$
\begin{aligned}
f_{i}^{n}(t) & =g_{i}^{n}(t) e^{-\int_{0}^{t} g_{i}^{n}(w) d w} \quad \text { if } 0<t<\tau_{3} \\
& =0 \quad \text { otherwise, } \\
f_{i}^{m}(t) & =g_{i}^{m}(t) e^{-\int_{0}^{t} g_{i}^{m}(w) d w} \quad \text { if } 0<t<\tau_{4} \\
& =0 \quad \text { otherwise }
\end{aligned}
$$

and the shift operator $\Delta$ is defined by

$$
\Delta^{a} x_{i}(t):=x_{i}(t-a)
$$

The functions $f_{i}^{p}, f_{i}^{l}, f_{i}^{n}$ and $f_{i}^{m}$ are density functions, i.e. $\int_{0}^{\tau_{i}^{1}} f_{i}^{p}(t) d t=1, \int_{0}^{\tau_{i}^{2}} f_{i}^{l}(t) d t=1, \int_{0}^{\tau_{i}^{3}} f_{i}^{n}(t) d t=1$, and $\int_{0}^{\tau_{i}^{4}} f_{i}^{m}(t) d t=1$.

Finally, integrating (3) and the last equation in (1) with respect to the age variable $a$, between $a=0$ and $a=+\infty$ one obtains the behavior of immature cells is represented by

$$
\begin{aligned}
\dot{x}_{i}(t)= & -\left(\delta_{i}+\beta_{i}\left(x_{i}(t)\right)\right) x_{i}(t) \\
& +L_{i} \int_{0}^{\tau_{i}^{4}} g_{i}^{m}(a) m_{i}(t, a) d a \\
& +u_{i-1}(t)
\end{aligned}
$$

where $u_{i-1}(t)=2 K_{i-1} \int_{0}^{\tau_{i-1}^{4}} g_{i-1}^{m}(a) m_{i-1}(t, a) d a$

$$
\begin{aligned}
\dot{\tilde{x}}_{i}(t)= & -\tilde{\beta}_{i}\left(\tilde{x}_{i}(t)\right) \tilde{x}_{i}(t) \\
& +\tilde{L}_{i} \int_{0}^{\tau_{4}} g_{i}^{m}\left(\theta_{1}\right) m_{i}\left(t, \theta_{1}\right) d \theta_{1}
\end{aligned}
$$

where

$$
m_{i}(t, a)=m_{i}(t-a, 0) e^{-\int_{0}^{a}\left(\gamma_{i}^{4}+g_{m}^{i}(w)\right) d w .}
$$

Equations (12) and (13) depend explicitly on each other, because of the term $m_{i}(t-a, 0)$, which contains the expressions $\Delta x_{i}^{a+\theta_{1}+\theta_{2}+\theta_{3}}(t)$ and $\Delta y_{i}^{a+\theta_{1}+\theta_{2}+\theta_{3}}(t)$.

If we define $h_{i}^{1}(t):=f_{i}^{p}(t) e^{-\gamma_{i}^{1} t}, h_{i}^{2}(t):=f_{i}^{l}(t) e^{-\gamma_{i}^{2} t}$, $h_{i}^{3}(t):=f_{i}^{n}(t) e^{-\gamma_{i}^{3} t}$ and $h_{i}^{4}(t):=f_{i}^{m}(t) e^{-\gamma_{i}^{4} t}$, the equations (12) and (13) can be rewritten as

$$
\begin{aligned}
\dot{x}_{i}(t)= & -\left(\delta_{i}+\beta_{i}\left(x_{i}(t)\right)\right) x_{i}(t) \\
& +L_{i} \cdot h_{i}^{4}(t) *\left(h_{i}^{3}(t) *\left[h_{i}^{2}(t) *\left\{h_{i}^{1}(t) * \omega_{i}(t)\right\}\right]\right) \\
& +u_{i-1}(t)
\end{aligned}
$$

$$
\begin{aligned}
\dot{\tilde{x}}_{i}(t)= & -\tilde{\beta}_{i}\left(\tilde{x}_{i}(t)\right) \tilde{x}_{i}(t) \\
& +\tilde{L}_{i} \cdot h_{i}^{4}(t) *\left(h_{i}^{3}(t) *\left[h_{i}^{2}(t) *\left\{h_{i}^{1}(t) * \omega_{i}(t)\right\}\right]\right)
\end{aligned}
$$

where $\omega_{i}(t):=\beta_{i}\left(x_{i}(t)\right) x_{i}(t)+\tilde{\beta}_{i}\left(\tilde{x}_{i}(t)\right) \tilde{x}_{i}(t)$

and $*$ denotes the usual convolution operator.

\section{ANALYSIS OF THE MODEL}

\subsection{Equilibrium points}

Let us denote by $x_{i}^{e}$ and $\tilde{x}_{i}^{e}$,for every $i$, the equilibrium points of (12) and (13), respectively; namely, the trajectories that satisfy $\frac{d x_{i}^{e}}{d t}=0$ and $\frac{d \tilde{x}_{i}^{e}}{d t}=0$. The $i t h$ equilibrium point is solution of the following algebraic system:

$$
\begin{aligned}
-\bar{u}_{i-1}= & -\left(1-L_{i} H_{i}(0)\right) \beta_{i}\left(x_{i}^{e}\right) x_{i}^{e}-\delta_{i} x_{i}^{e} \\
& +L_{i} H_{i}(0) \tilde{\beta}_{i}\left(\tilde{x}_{i}^{e}\right) \tilde{x}_{i}^{e} \\
0= & \tilde{L}_{i} H_{i}(0) \beta_{i}\left(x_{i}^{e}\right) x_{i}^{e} \\
& -\left(1-\tilde{L}_{i} H_{i}(0)\right) \tilde{\beta}_{i}\left(\tilde{x}_{i}^{e}\right) \tilde{x}_{i}^{e}
\end{aligned}
$$

where

$\bar{u}_{i-1}= \begin{cases}0 & \text { if } i=1 \\ 2 K_{i-1} h_{i-1} & \text { if } i>1 \\ \cdot\left(\beta_{i-1}\left(x_{i-1}^{e}\right) x_{i-1}^{e}+\tilde{\beta}_{i-1}\left(\tilde{x}_{i-1}^{e}\right) \tilde{x}_{i-1}^{e}\right) & \end{cases}$ and

$$
H_{i}(s)=H_{i}^{1}(s) \cdot H_{i}^{2}(s) \cdot H_{i}^{3}(s) \cdot H_{i}^{4}(s)
$$

with $H_{i}^{1}(s)=\int_{0}^{\tau_{i}^{1}} h_{i}^{1}(t) e^{-s t} d t, H_{i}^{2}(s)=\int_{0}^{\tau_{i}^{2}} h_{i}^{2}(t) e^{-s t} d t$, $H_{i}^{3}(s)=\int_{0}^{\tau_{i}^{3}} h_{i}^{3}(t) e^{-s t} d t$ and $H_{i}^{4}(s)=\int_{0}^{\tau_{i}^{4}} h_{i}^{4}(t) e^{-s t} d t$.

We can readily note that the points $x_{i}^{e}=0$ and $\tilde{x}_{i}^{e}=0$ satisfy (14) and (15). We will refer to this equilibrium point as the trivial equilibrium point. From (14) and (15), a non-trivial equilibrium point satisfy

$$
\begin{aligned}
& \beta_{i}\left(x_{i}^{e}\right)= \begin{cases}\frac{\delta_{1}}{\alpha_{1}} & \text { if } i=1 \\
\frac{\delta_{i}}{\alpha_{i}}-\left(\frac{\bar{u}_{i-1}}{\alpha_{i}}\right) \frac{1}{x_{i}^{e}} & \text { if } i>1\end{cases} \\
& \tilde{\beta}_{i}\left(\tilde{x}_{i}^{e}\right)= \begin{cases}\left(\frac{\delta_{1} x_{i}^{e}}{\tilde{\alpha}_{1}}\right) \frac{1}{\tilde{x}_{1}^{e}} & \text { if } i=1 \\
\left(\frac{\delta_{i} x_{i}^{e}-\bar{u}_{i-1}}{\tilde{\alpha}_{i}}\right) \frac{1}{\tilde{x}_{i}^{e}} & \text { if } i>1\end{cases}
\end{aligned}
$$

where

$$
\alpha_{i}:=\frac{2\left(1-K_{i}\right) H_{i}(0)-1}{1-2\left(1-\sigma_{1}\right)\left(1-K_{i}\right) H_{i}(0)}
$$

and

$$
\tilde{\alpha}_{i}:=\frac{2\left(1-K_{i}\right) H_{i}(0)-1}{2\left(1-\sigma_{1}\right)\left(1-K_{i}\right) H_{i}(0)}
$$

recall $L_{i}:=2 \sigma_{i}\left(1-K_{i}\right), \tilde{L}_{i}:=2\left(1-\sigma_{i}\right)\left(1-K_{i}\right)$.

The next proposition deals with existence and uniqueness of positive equilibrium points $x_{i}^{e}$.

Proposition 1. If $1<2\left(1-K_{i}\right) H_{i}(0)<\frac{1}{1-\sigma_{i}}$ for all $i$, and $\beta_{1}(0)>\frac{\delta_{1}}{\alpha_{1}}$ then we have a unique positive equilibrium point $x_{i}^{e}$.

Proof. First note that $\alpha_{i}$ is non negative for every $i$ if our assumption is satisfied. For $i=1, \beta_{1}\left(x_{1}^{e}\right)=\frac{\delta_{1}}{\alpha_{1}}$; the existence and uniqueness is guaranteed if $\beta_{1}(0)>\frac{\delta_{1}}{\alpha_{1}}$. For $i \geq 2$, let $\psi_{i}: \mathbb{R}_{+} \backslash\{0\} \rightarrow \mathbb{R}$ be given by $\psi_{i}(\bar{x})=\frac{\delta_{i}}{\alpha_{i}}-\frac{b_{i}}{\alpha_{i}} \frac{1}{\bar{x}}$. The non-negativeness of $\alpha_{i}$ implies that the function $\psi_{i}$ is strictly increasing with respect to $\bar{x}\left(\frac{d}{d \bar{x}} \psi_{i}(\bar{x})>0\right)$, $\lim _{\bar{x} \rightarrow+\infty} \psi_{i}(\bar{x})=\frac{\delta_{i}}{\alpha_{i}}$ is positive and $\lim _{\bar{x} \rightarrow 0-} \psi_{i}(\bar{x})=-\infty$. The functions $\beta_{i}$ and $\psi_{i}$ are continuous over $\mathbb{R}_{+} \backslash\{0\}$ and their difference $\left(\beta_{i}-\psi_{i}\right)$ is a strictly decreasing function, we have that $\lim _{\bar{x} \rightarrow 0^{-}}\left(\beta_{i}(\bar{x})-\psi_{i}(\bar{x})\right)=\infty$ and 
$\lim _{\bar{x} \rightarrow+\infty}\left(\beta_{i}(\bar{x})-\psi_{i}(\bar{x})\right)=-\frac{\delta_{i}}{\alpha_{i}}$. This implies that there is a positive real number $x^{*}$ such that $\beta_{i}\left(x^{*}\right)-\psi_{i}\left(x^{*}\right)=0$. In other words, (14) has a unique positive solution for every $i$.

As $\tilde{\beta}_{i}\left(\tilde{x}_{i}^{e}\right)=\frac{1}{\tilde{x}_{i}^{e}} \frac{2\left(1-\sigma_{i}\right)\left(1-K_{i}\right) H_{i}(0)}{\left(1-2\left(1-\sigma_{i}\right)\left(1-K_{i}\right) H_{i}(0)\right)} \beta_{i}\left(x_{i}^{e}\right) x_{i}^{e}$, it is easy to see that for a suitable function $\tilde{\beta}_{i}$, there will be a unique intersection point between the functions $x \mapsto \tilde{\beta}_{i}\left(\tilde{x}_{i}^{e}\right)$ and $x \mapsto \frac{1}{\tilde{x}_{i}^{e}} \frac{2\left(1-\sigma_{i}\right)\left(1-K_{i}\right) H_{i}(0)}{\left(1-2\left(1-\sigma_{i}\right)\left(1-K_{i}\right) H_{i}(0)\right)} \beta_{i}\left(x_{i}^{e}\right) x_{i}^{e}$.

In the following, we analyze the asymptotic stability of (12) and (13) by studying the behaviour of their steady states.

\subsection{Model linearization and stability}

Let us define a perturbed trajectory of the equilibrium points of (12) and (13) by $X_{i}(t):=x_{i}(t)-x_{i}^{e}(t)$ and $\tilde{X}_{i}(t):=\tilde{x}_{i}(t)-\tilde{x}_{i}^{e}(t)$, for every $i$. The linearization of (12) and (13) around their equilibrum points is

$$
\begin{aligned}
\frac{d}{d t} X_{i}(t)= & -\left(\delta_{i}+\mu_{i}\right) X_{i}(t) \\
& +L_{i} \mu_{i} \cdot\left[h_{i}^{4} *\left(h_{i}^{3} *\left(h_{i}^{2} *\left(h_{i}^{1} * X_{i}\right)\right)\right)\right](t) \\
& +L_{i} \tilde{\mu}_{i} \cdot\left[h_{i}^{4} *\left(h_{i}^{3} *\left(h_{i}^{2} *\left(h_{i}^{1} * \tilde{X}_{i}\right)\right)\right)\right](t) \\
& +2 K_{i-1} \mu_{i-1} \\
& \cdot\left[h_{i-1}^{4} *\left(h_{i-1}^{3} *\left(h_{i-1}^{2} *\left(h_{i-1}^{1} * X_{i-1}\right)\right)\right)\right](t) \\
& +2 K_{i-1} \tilde{\mu}_{i-1} \\
& \cdot\left[h_{i}^{4} *\left(h_{i}^{3} *\left(h_{i}^{2} *\left(h_{i}^{1} * \tilde{X}_{i-1}\right)\right)\right)\right](t)
\end{aligned}
$$

and

$$
\begin{aligned}
\frac{d}{d t} \tilde{X}_{i}(t)= & -\tilde{\mu}_{i} \tilde{X}_{i}(t) \\
& +\tilde{L}_{i} \mu_{i} \cdot\left[h_{i}^{4} *\left(h_{i}^{3} *\left(h_{i}^{2} *\left(h_{i}^{1} * X_{i}\right)\right)\right)\right](t) \\
& +\tilde{L}_{i} \tilde{\mu}_{i} \cdot\left[h_{i}^{4} *\left(h_{i}^{3} *\left(h_{i}^{2} *\left(h_{i}^{1} * \tilde{X}_{i}\right)\right)\right)\right](t)
\end{aligned}
$$

where

$$
\begin{aligned}
\mu_{i} & =\left.\frac{d}{d x}\left(\beta_{i}(x) x\right)\right|_{x=x_{i}} \\
\tilde{\mu}_{i} & =\left.\frac{d}{d x}\left(\tilde{\beta}_{i}(x) x\right)\right|_{x=\tilde{x}_{i}}
\end{aligned}
$$

Taking the Laplace transform of (18) and (19), we can see that the characteristic equation of the system represented by (18) and (19) is given by

$$
\prod_{i=1}^{n} A_{i}(s)=0
$$

where $A_{i}(s)=d_{i}^{11}(s) d_{i}^{22}(s)-d_{i}^{12}(s) d_{i}^{21}(s)$ with

$$
\begin{gathered}
d_{i}^{11}(s)=s+\delta_{i}+\mu_{i}-L_{i} \mu_{i} H_{i}(s), \\
d_{i}^{12}(s)=-L_{i} \tilde{\mu}_{i} H_{i}(s), \\
d_{i}^{21}(s)=-\tilde{L}_{i} \mu_{i} H_{i}(s), \\
d_{i}^{22}(s)=s+\tilde{\mu}_{i}-\tilde{L}_{i} \tilde{\mu}_{i} H_{i}(s)
\end{gathered}
$$

It is a simple exercise to see that each $A_{i}(s)$ can be expressed in the form

$$
\begin{aligned}
A_{i}(s)= & \left(s+\tilde{\mu}_{i}\right)\left(s+\delta_{i}+\mu_{i}\right) \\
& \cdot\left(1-\frac{L_{i} \mu_{i} H_{i}(s)}{\left(s+\delta_{i}+\mu_{i}\right)}\left(1+\frac{\tilde{L}_{i} \tilde{\mu}_{i}\left(s+\delta_{i}+\mu_{i}\right)}{L_{i} \mu_{i}\left(s+\tilde{\mu}_{i}\right)}\right)\right)
\end{aligned}
$$

If $\tilde{\mu}_{i}>0$ and $\delta_{i}+\mu_{i}>0$ then, by the observation that each $H_{i}(s)$ is $H_{\infty}$-stable, the system is stable if and only if the roots of

$$
1-\frac{L_{i} \mu_{i} H_{i}(s)}{\left(s+\delta_{i}+\mu_{i}\right)}\left(1+\frac{\tilde{L}_{i} \tilde{\mu}_{i}\left(s+\delta_{i}+\mu_{i}\right)}{L_{i} \mu_{i}\left(s+\tilde{\mu}_{i}\right)}\right)=0
$$

are in the open left half plane, for all $i$. Note that the characteristic equation studied in Ozbay et al. (2012) was in the form

$$
1-\frac{L_{i} \mu_{i} H_{i}(s)}{\left(s+\delta_{i}+\mu_{i}\right)}=0
$$

Therefore, (21) is a generalization of (22). Since the factor

$$
\left(1+\frac{\tilde{L}_{i} \tilde{\mu}_{i}\left(s+\delta_{i}+\mu_{i}\right)}{L_{i} \mu_{i}\left(s+\tilde{\mu}_{i}\right)}\right)
$$

is a finite dimensional (in fact first order) perturbation of the unit 1 , we are able to derive stability properties depending on the parameters of this system, using the techniques employed in Ozbay et al. (2012).

\section{CONCLUSIONS}

In this paper, we have proposed a new model for the dynamical cell behavior in AML. First, we have started with the PDE's representing the cell dynamics for the phases $G_{0}, G_{1}, S, G_{2}$ and $M$. Then, by analyzing the solutions of these PDE's, the model has been transformed into a form of two coupled nonlinear systems involving distributed delays. An equilibrium analysis is done and characteristic equation for the linearized system is obtained. Thus, the problem at hand is put into the framework of the earlier work, Ozbay et al. (2012) whose stability results can be extended to the more refined model considered here. Currently, experiments conducted using biological data are performed in order to estimate the parameters of this model.

\section{REFERENCES}

Adimy, M., Crauste, F., and El Abdllaoui, A. (2008). Discrete maturity-structured model of cell differentiation with applications to acute myelogenous leukemia. $J$. Biological Systems, 16, No. 3, 395-424.

Adimy, M., Crauste, F., and Marquet C. (2010). Asymptotic behavior and stability switch for a matureimmature model of cell differentiation. Nonlinear Analysis: Real World Applications, 11, No. 4, 2913-2929.

Dingli, D., and Pacheco, J. M. (2010). Modeling the architecture and dynamics of hematopoiesis. Wiley Interdisciplinary Reviews: Systems Biology and Medicine, 2, No. 2, 235-244.

Foley, C., and Mackey, M.C. (2009). Dynamic hematological disease: a review. J. Mathematical Biology, 58, No. 12, 285-322.

Mackey, M. C. (1978). Unified hypothesis for the origin of aplastic anaemia and periodic hematopoiesis. Blood, 51 , No. 5, 941-956.

Niculescu, S-I., Kim, P. S., Gu, K., Lee, P.P., and Levy, D. (2010). Stability crossing boundaries of delay systems 
modeling immune dynamics in leukemia. Discrete and Continuous Dynamical Systems Series B, 13, No. 1, 129156.

Özbay, H., Bonnet, C.,Benjelloun, H., and Clairambault, J. (2012). Stability Analysis of Cell Dynamics in Leukemia. Mathematical Modelling of Natural Phenomena, 7, No. 1, pp. 203-234.

Özbay, H., Benjelloun, H., Bonnet, C., Clairambault, J. (2010). Stability conditions for a system modeling cell dynamics in leukemia. Preprints of IFAC Workshop on Time Delay Systems, TDS2010, Prague, Czech Republic, June 2010.

Perthame, B. (2007). Transport equations in biology. Frontiers in Mathematics, Birkhäuser Verlag..

Rowe, J. (2008). Why is clinical progress in acute myelogenous leukemia so slow? Best Practice 8 Research Clinical Haematology, vol. 21, No. 1, pp. 1-3. 